Supporting Information

\title{
High Energy Aqueous Rechargeable Nickel Zinc Battery Employing Hierarchical NiV-LDH Nanosheet-built Microspheres on Reduced Graphene Oxide
}

\author{
Mohadese Rastgoo-Deylami, Ali Esfandiar* \\ Department of Physics, Sharif University of Technology, Tehran, 11155-9161, Iran \\ ${ }^{*}$ Corresponding authors \\ E-mail: esfandiar@physics.sharif.edu
}




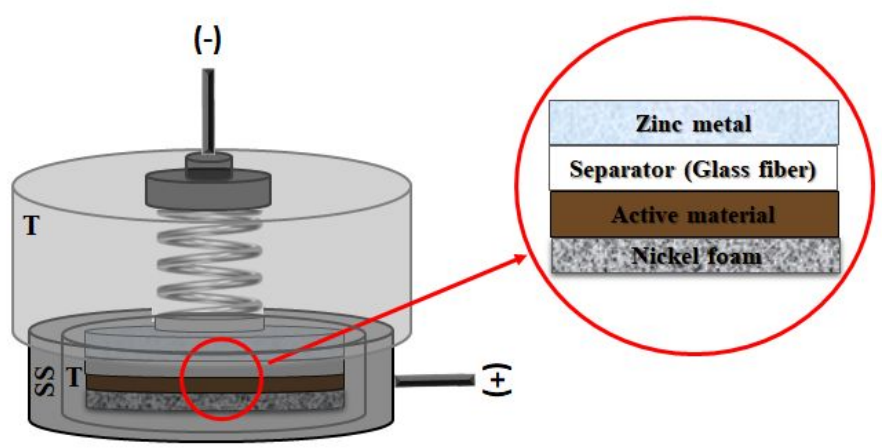

T: Teflon

SS: Stainless Steel

Figure S1. Schematic diagram of the homemade electrochemical cell used in this study.



Figure S2. TEM image of Multiwall carbon nanotube (MW-CNT) used in this work. 

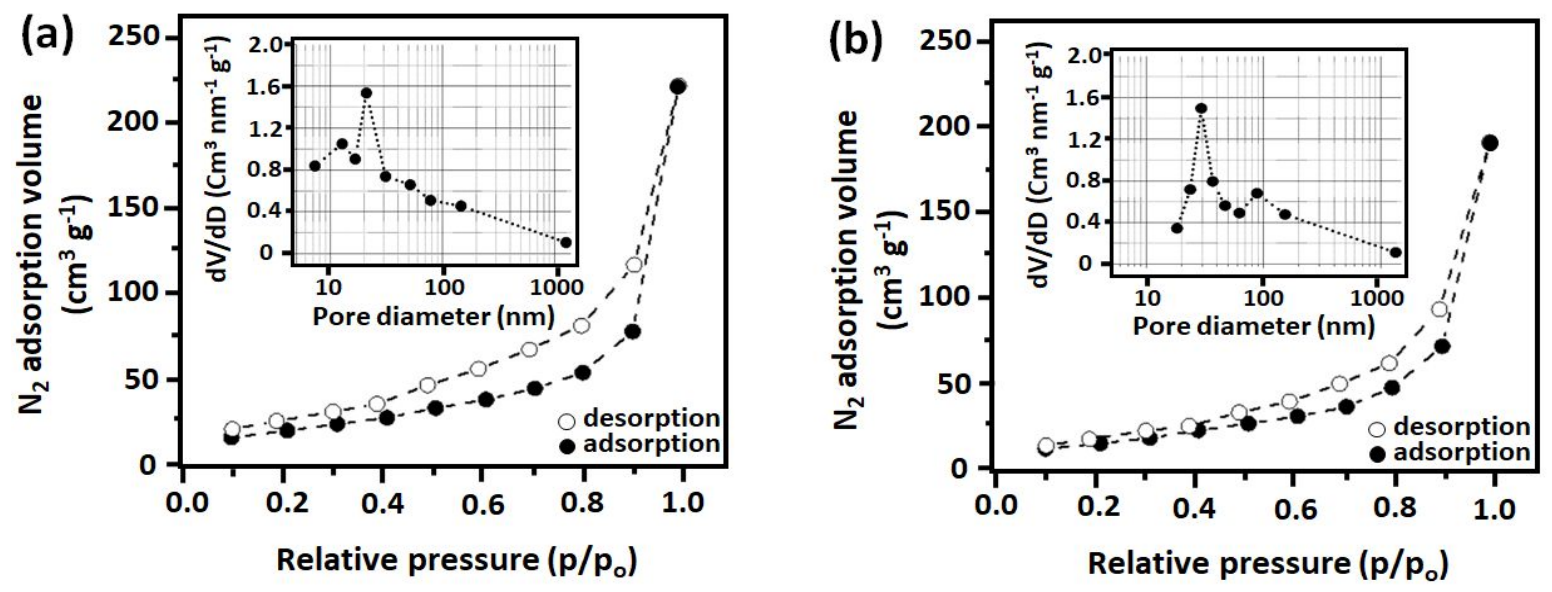

Figure S3. $\mathrm{N}_{2}$ adsorption/ desorption isotherms and $\mathrm{BJH}$ pore size distribution (inside figures) of (a) the NVGO and (b) NVCNT samples.


Figure S4. Thermogravimetric analysis (TGA) of (a) NV, (b) NVGO and (c) NVCNT samples under $\mathrm{N}_{2}$ flowing atmosphere with the temperature rate of $5{ }^{\circ} \mathrm{C} \mathrm{min}^{-1}$. 

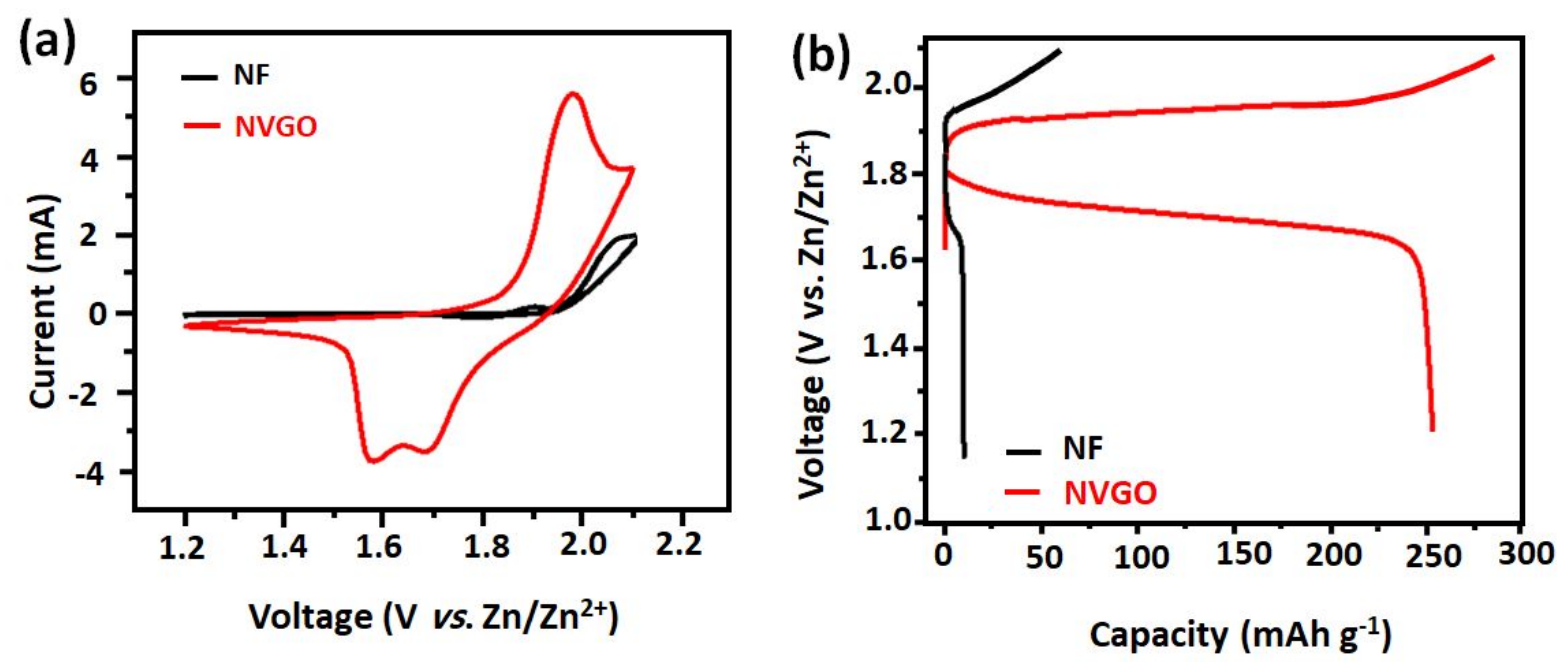

Figure S5. (a) Cyclic voltammogram of Nickel foam (NF) and NVGO electrodes at $0.5 \mathrm{mV} \mathrm{s}^{-1}$ in the voltage range of 1.2 to $2.1 \mathrm{~V} v s$. $\mathrm{Zn} / \mathrm{Zn}^{2+}$. (b) Charge and discharge profiles of $\mathrm{NF}$ and NVGO electrodes with current density of $0.5 \mathrm{~A} \mathrm{~g}^{-1}$. The mentioned analyses are conducted at room temperature in $\mathrm{KOH}(6 \mathrm{M})$ saturated with $\mathrm{ZnO}$ powder as electrolyte.

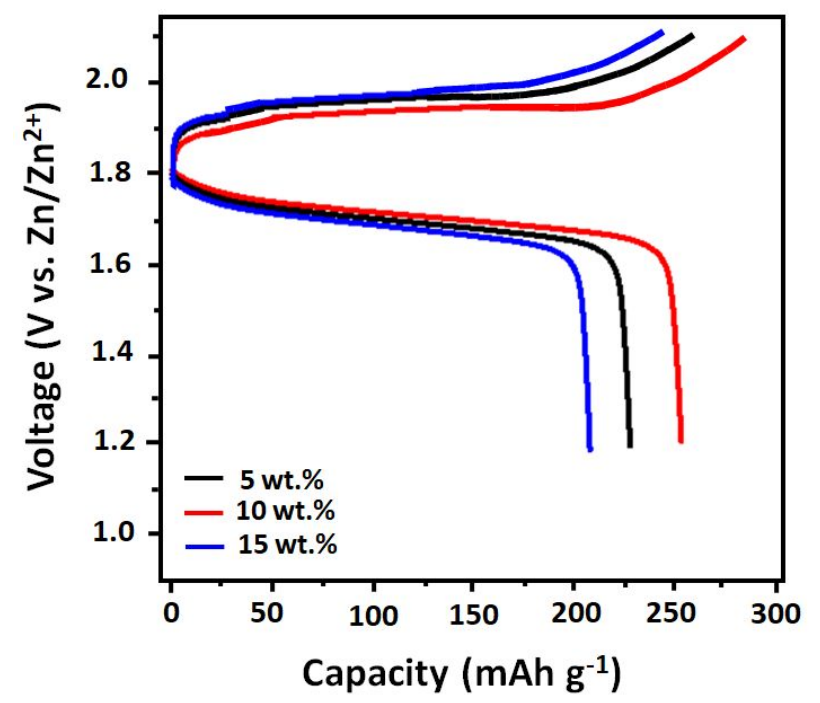

Figure S6. Charge and discharge profiles of NVGO electrodes including of different amounts of $\mathrm{GO}(5,10$ and $15 \mathrm{wt} . \%)$, in the voltage range of 1.2 to $2.1 \mathrm{~V} v s . \mathrm{Zn} / \mathrm{Zn}^{2+}$ with $0.5 \mathrm{~A} \mathrm{~g}^{-1}$ at room temperature. 


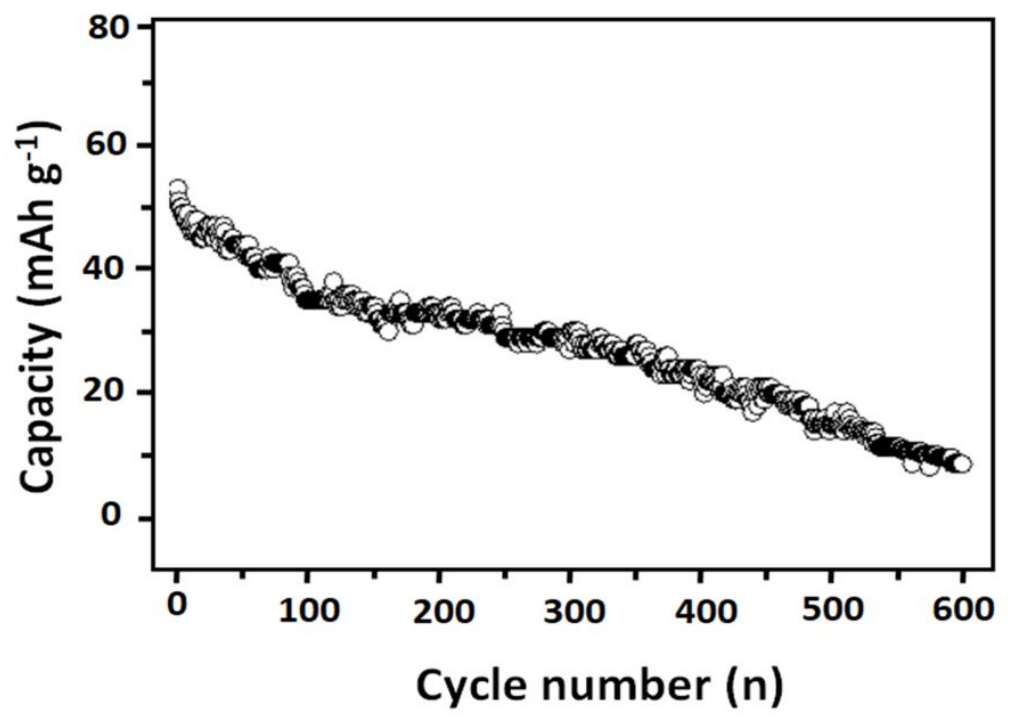

Figure S7. The cycling performance of the NV sample with current density of $4.5 \mathrm{~A} \mathrm{~g}^{-1}$ for 600 cycles at room temperature.
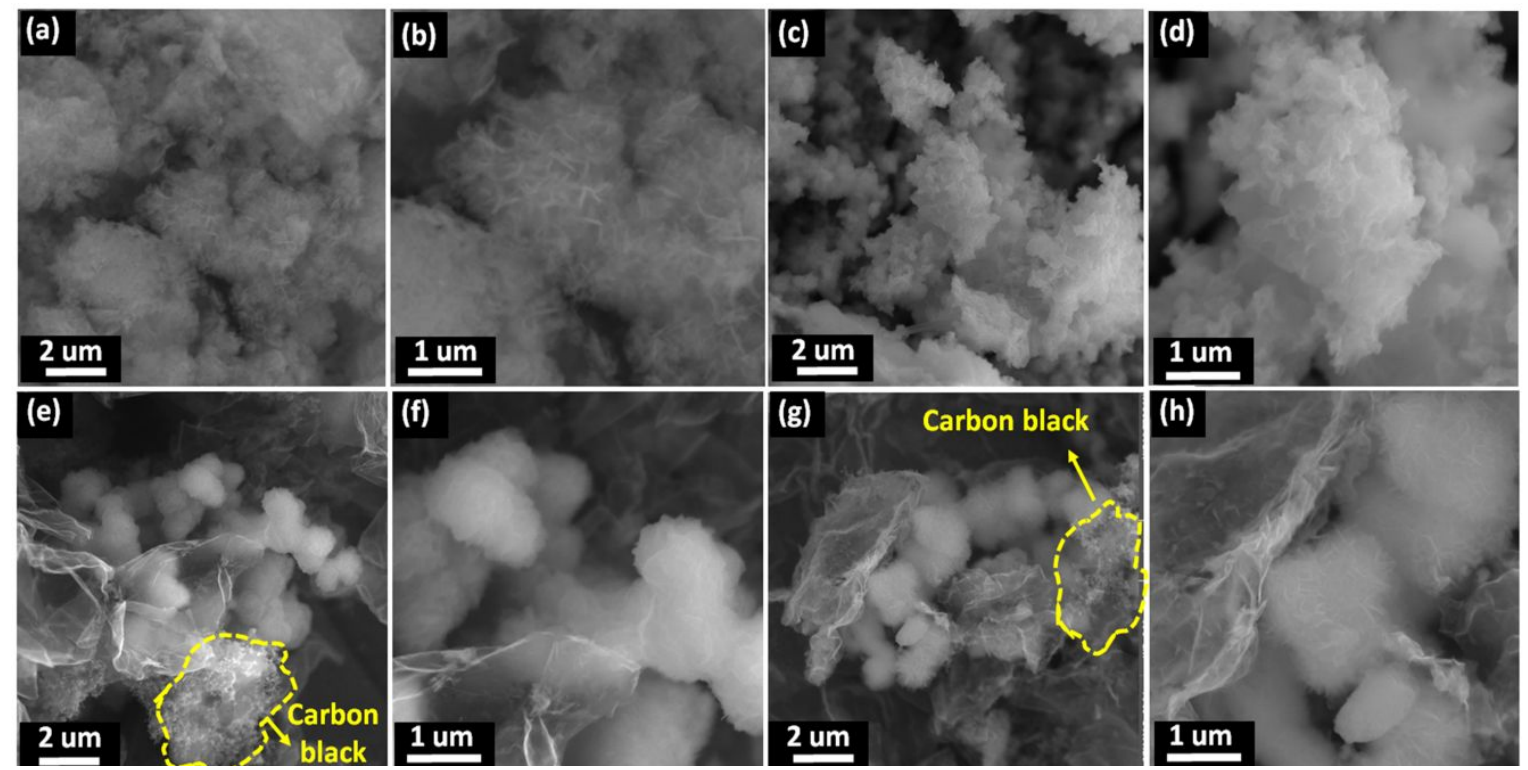

Figure S8. The SEM images of $(a, b)$ the initial and (c, d) cycled NV electrode, and (e, f) initial and $(\mathrm{g}, \mathrm{h})$ cycled NVGO electrodes. 
Table S1. The XPS results of the fitted Nickel and Vanadium spectra for the NVGO sample.

\begin{tabular}{ccccc}
\hline XPS peaks & Binding Energy $(\mathrm{eV})$ & FWHM & Peak area & $\begin{array}{c}\text { Molar ratio } \\
(\mathrm{Ni} / \mathrm{V})\end{array}$ \\
\hline $\mathrm{Ni} 2 \mathrm{p}_{3 / 2}$ & 855.9 & 2.91 & 2636.20 & \\
\hline $\mathrm{Ni} 2 \mathrm{p}_{1 / 2}$ & 874.1 & 3.40 & 1391.39 & 1.52 \\
\hline $\mathrm{V} 2 \mathrm{p}_{3 / 2}$ & 517.1 & 2.41 & 910.40 & \\
\hline $\mathrm{V} 2 \mathrm{p}_{1 / 2}$ & 524.2 & 1.86 & 123.89 & \\
\hline
\end{tabular}

To calculate the molar ratio of Ni to V, Relative Sensitivity Factors (RSF) for the Ni2 $\mathrm{p}_{3 / 2}$ and $\mathrm{V} 2 \mathrm{p}_{3 / 2}$ are considered as 2.696 and 1.411 , respectively. 
Table S2. Comparison of electrochemical performances of active materials used in rechargeable Ni-Zn battery, previously published in the literatures.

\begin{tabular}{|c|c|c|c|c|c|c|c|c|}
\hline Electrodes & $\begin{array}{c}\text { Voltage } \\
\left(\mathrm{V} v s . \mathrm{Zn} / \mathbf{Z n}^{2+}\right)\end{array}$ & $\begin{array}{l}\text { Capacity } \\
\left(\mathrm{mAh} \mathrm{g} \mathrm{g}^{-1}\right)\end{array}$ & $\begin{array}{c}\text { Capacity } \\
\left(\mathrm{mAh} \mathrm{Cm}^{-2}\right)\end{array}$ & $\begin{array}{c}\text { Energy } \\
\text { density } \\
\left(\mathbf{W h ~ k g}^{-1}\right)\end{array}$ & $\begin{array}{c}\text { Power } \\
\left(W_{k g}^{-1}\right)\end{array}$ & $\begin{array}{l}\text { Areal energy } \\
\text { density } \\
\left(\mathrm{mWh} \mathbf{C m}^{-2}\right)\end{array}$ & 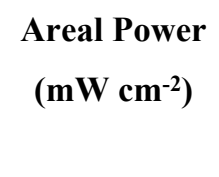 & Ref \\
\hline $\mathrm{Co}_{3} \mathrm{O}_{4} @ \mathrm{NiV} \mathrm{LDH} / \mathrm{Zn}$ & 1.71 & $180\left(0.45 \mathrm{~A} \mathrm{~g}^{-1}\right)$ & $1.98\left(5 \mathrm{~mA} \mathrm{~cm}^{-2}\right)$ & 143.5 & 1140 & 2.2 & 17.1 & {$[14]$} \\
\hline $\mathrm{Ni}_{2} \mathbf{P} / \mathrm{Zn}$ & 1.78 & $231\left(1 \mathrm{~A} \mathrm{~g}^{-1}\right)$ & & 318 & 1370 & & & {$[35]$} \\
\hline $\mathbf{N i}_{3} \mathbf{S}_{2} @ \mathbf{P A N I} / \mathbf{Z n}$ & & $242.8 \pm 8.8\left(5.1 \mathrm{~A} \mathrm{~g}^{-1}\right)$ & & 386.7 & 38800 & & & {$[46]$} \\
\hline Co-doped $\mathrm{Ni}(\mathrm{OH})_{2} / \mathrm{Zn}$ & 1.75 & $346\left(5 \mathrm{~A} \mathrm{~g}^{-1}\right)$ & & 148.54 & 1725 & & & [11] \\
\hline $\mathrm{NiCo}_{2} \mathrm{O}_{4}$ nanosheets $/ \mathrm{Zn}$ & & $183.1\left(1.6 \mathrm{~A} \mathrm{~g}^{-1}\right)$ & & 303.8 & 49000 & & & {$[36]$} \\
\hline $\mathrm{NiO} / \mathrm{Zn}$ & 1.75 & $155\left(1 \mathrm{~A} \mathrm{~g}^{-1}\right)$ & & 228 & 935 & & & {$[10]$} \\
\hline $\mathrm{Co}_{3} \mathrm{O}_{4} / \mathrm{Zn}$ & 1.78 & $162\left(1 \mathrm{Ag}^{-1}\right)$ & & 241 & 1506 & & & {$[37]$} \\
\hline $\mathrm{Co}_{3} \mathrm{O}_{4} @ \mathrm{NiO} / \mathrm{Zn}$ & 1.70 & 182.6 & $2.89\left(5 \mathrm{~mA} \mathrm{~cm}{ }^{-2}\right)$ & 215.51 & 3450 & 5.12 & 82.21 & {$[38]$} \\
\hline $\mathrm{Ni}_{3} \mathrm{~S}_{2} @ \mathbf{C} / \mathbf{Z n}$ & & $234.3\left(6.9 \mathrm{~A} \mathrm{~g}^{-1}\right)$ & & 356 & 58600 & & & [39] \\
\hline NiAICO@CNT /Zn & 1.75 & $354\left(6.7 \mathrm{~A} \mathrm{~g}^{-1}\right)$ & & 274 & 16000 & & & {$[13]$} \\
\hline $\mathrm{NiCoO}_{3} \mathrm{H}_{x} / \mathrm{Zn}$ & & & 5.04 & & & 0.12 & 32.8 & [40] \\
\hline $\mathrm{MnO}_{2} / \mathrm{Zn}$ & & $306\left(0.06 \mathrm{~A} \mathrm{~g}^{-1}\right)$ & & & & 6.18 & 148.2 & [41] \\
\hline $\mathrm{Li}_{4} \mathrm{Ti}_{5} \mathrm{O}_{12} / \mathrm{LiCoO}_{2}$ & 2.5 & & & & & 2.2 & 22 & {$[42]$} \\
\hline $\begin{array}{c}\mathrm{CC}-\mathrm{CF} @ \mathrm{NiO}^{*} / \mathrm{CC}- \\
\mathrm{CF} @ \mathrm{ZnO}\end{array}$ & 1.77 & & $0.39\left(0.5 \mathrm{~mA} \mathrm{~cm}^{-2}\right)$ & 355.7 & 17900 & & & [43] \\
\hline $\mathrm{NiCo}_{2} \mathrm{O}_{4} / \mathrm{Zn}$ & & $230.1\left(0.5 \mathrm{~A} \mathrm{~g}^{-1}\right)$ & & 301.3 & 1309 & & & [44] \\
\hline $\mathrm{NiS}_{2} @ \mathrm{rGO} / \mathrm{Zn}$ & 1.75 & $209.4\left(1 \mathrm{~A} \mathrm{~g}^{-1}\right)$ & & 357.7 & 1750 & & & [45] \\
\hline$\overline{\mathrm{FeO}_{\mathrm{x}} / \mathrm{Ni}(\mathrm{OH})_{2}}$ & & $126\left(1.5 \mathrm{~A} \mathrm{~g}^{-1}\right)$ & & 120 & 15000 & 0.35 & 40 & [47] \\
\hline $\begin{array}{c}\mathrm{Co}_{\mathbf{x}} \mathrm{Fe}_{3-\mathrm{x}} \mathrm{O}_{4} \\
/ \mathrm{Co}_{3} \mathrm{O}_{4} @ \mathrm{NiCOO}\end{array}$ & & & 2.13 & 132.55 & 310 & 2.1 & 42.8 & {$[48]$} \\
\hline NiV LDH with 10 wt.\% & 1.76 & $253.9\left(0.5 \mathrm{~A} \mathrm{~g}^{-1}\right)$ & $3.78\left(7.5 \mathrm{~mA} \mathrm{~cm}^{-2}\right)$ & 447.63 & 492.4 & 6.66 & 7.33 & This \\
\hline GO $/ \mathbf{Z n}$ & & 195.6 $\left(4.5 \mathrm{~A} \mathrm{~g}^{-1}\right)$ & $2.91\left(67 \mathrm{~mA} \mathrm{~cm}^{-2}\right)$ & 368.15 & 2890 & 5.18 & 46.88 & work \\
\hline
\end{tabular}

